\title{
Hierarchical Attribute Based Revocable Data Access Control For Multi Authority Cloud Storage
}

\author{
${ }^{*}$ Mr. B. Gopi Krishna ${ }^{1}$, Mr.B. Siva Rama Krishna ${ }^{2}$ \\ ${ }^{I} M$. Tech in Dept. of Computer Science and Engineering, LBRCE College, Mylavaram, India. \\ ${ }^{2}$ Assistant professor in Dept. of Computer Science and Engineering, LBRCE College, Mylavaram, \\ India. \\ Corresponding Author: Mr. B. Gopi Krishna
}

\begin{abstract}
Distributed computing is rising colossally because of its points of interest and the adaptable stockpiling administrations given by it. Because of this the quantity of clients has come to at the top. Clearly the clients will be sharing the touchy information through the cloud. Also, the client can't trust the untrusted cloud server. Subsequently the information get to control has turned out to be exceptionally testing in distributed storage framework. In existing work revocable information get to control plan is proposed for multi-expert distributed storage frameworks which bolsters the get to control in light of the specialist control. The approved clients who have qualified traits given by different specialists can get to the information. However, it couldn't control the assaults which can happen by the approved client who are not having qualified characteristics. In this work we propose another calculation Improved Security Data Access Control which Overcomes the issue exists in the current work. And furthermore incorporate the effective characteristic renouncement technique for multi specialist distributed storage.
\end{abstract}

Indexterms: Access control, multi-authority, efficient CP-ABE, attribute revocation, cloud storage.

\section{Introduction}

Like Cloud Computing, Cloud Storage has likewise been developing in fame as of late because of a considerable lot of an indistinguishable reasons from Cloud Computing. Distributed storage conveys virtualized capacity on request, over a system in light of a demand for a given nature of administration (QoS). There is no need to buy stockpiling or at times even arrangement it before putting away information. Distributed storage is a vital bundle of distributed computing, which offers agreements for cloud information sellers to have their information in the cloud. This new model of information facilitating and get to administrations acquaints an awesome test with information get to control. Since the cloud information sellers can't be completely trust on cloud server and they are not prepared to trust on servers to control the information get to Ciphertext - Policy Attribute Based Encryption is seen as a standout amongst the most proper innovations for information get to control in distributed storage frameworks, in light of the fact that if offers more straightforward get to control approaches and systems to the cloud information merchants. In CP-ABE conspire, there is a particular specialist that is in charge of characteristic administration, key era, key exchange and key appropriations [1], [3]. The expert can be the enlisted office situated in various areas. The cloud information sellers can express the get to techniques and scramble the information as per the systems. Every client will be provided a mystery key imitating its properties. The information can be unscrambled the cloud clients by checking its properties in view of the get to techniques [1], [2].

\section{CP-ABE}

CP-ABE offers two sorts of frameworks: 1.Single Authority CP-ABE 2.Multi-Authority CPABE Single Authority CPABE: Attributes of the cloud information merchants are overseen by sole specialist. Broad research has accomplished for single specialist in distributed storage framework, a client may hold characteristics issued by numerous experts and the information proprietors may impart the information to the client figured out how to various experts which is an incredible test in single specialist [2], [9]. Multi-Authority CP-ABE: Attributes of the diverse areas and cloud 
information sellers are overseen by various experts. Multi-Authority CP-ABE is more able plan for information get to control of distributed storage frameworks, as clients may grip properties issued by various experts and information proprietors may likewise share the information utilizing access approach characterized over traits from various specialists. In this paper, we initially propose a Fortified multi-expert CPABE plot, where an expressive, productive and more secured disavowal technique is proposed to tackle the characteristic repudiation and mysterious information get to issues in the distributed storage framework. Effectiveness in calculation and quality repudiation are the basic necessities while outlining the get to control plans [2]. In Efficient Computation, there are three operations required in particular 1.Encryption 2.Decryption 3.Revocation In Efficient Attribute Revocation, there are two necessities 1.Backward Security 2.Forward Security In this paper, we plan another braced multi-expert $\mathrm{CP}-\mathrm{ABE}$ conspire with productive unscrambling and offer a proficient quality denial technique.

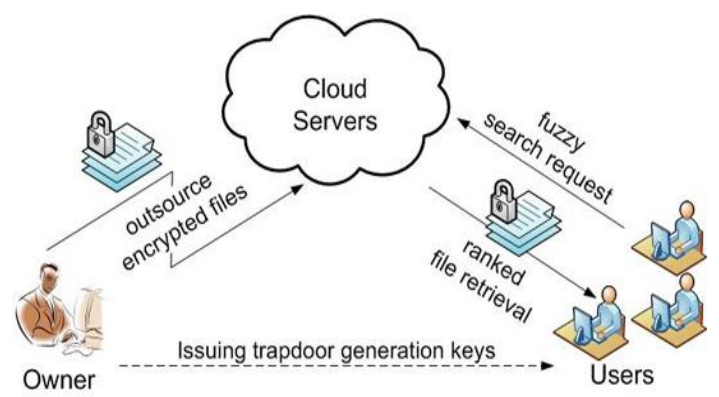

Fig 1: Privacy-assured and Effective Cloud Data Utilization.

This new worldview of information facilitating and information get to administrations acquaints an incredible test with information get to control. Since the cloud server can't be completely trusted by information proprietors, they can never again depend on servers to do get to control. Figure content Policy Attributebased Encryption (CP-ABE), is viewed as a standout amongst the most reasonable innovations for information get to control in distributed storage frameworks, since it gives the information proprietor more straightforward control on get to strategies. In CP-ABE conspire; there is an expert that is in charge of characteristic administration and key conveyance. The specialist can be the enlistment office in a college, the human asset division in an organization, and so on. The information proprietor characterizes the get to approaches and scrambles information as indicated by the arrangements. Every client will be issued a mystery key mirroring its characteristics. A client can unscramble the information just when its traits fulfill the get to strategies [9], [2]. There are two sorts of CP-ABE frameworks: single expert where all qualities are overseen by a solitary specialist, and multi-expert $\mathrm{CP}-\mathrm{ABE}$, where properties are from various areas and overseen by various specialists. For instance, in an E-wellbeing framework, information proprietors may share the information utilizing the get to strategy "Doctor AND Researcher", where the trait ""Doctor"" is issued by a therapeutic association and the property ""Researcher"" is issued by the executives of a clinical trial. Nonetheless, it is hard to straightforwardly apply these multi-expert CP-ABE plans to multiauthority distributed storage frameworks in view of the trait repudiation issue. In multi-specialist distributed storage frameworks, clients' characteristics can be changed powerfully. A client might be entitled some new characteristics or disavowed some present qualities. What's more, his consent of information get to ought to be changed as needs be. In any case, existing trait renouncement strategies either depend on a confided in server or absence of proficiency, they are not reasonable for managing the quality denial issue in information get to control in multi-expert distributed storage frameworks. In this paper, we initially propose a revocable multiauthority $\mathrm{CP}-\mathrm{ABE}$ conspire, where a productive and secure renouncement strategy is proposed to take care of the quality denial issue in the framework. As depicted our trait renouncement technique is productive as in it brings about less correspondence cost and calculation cost, and is secure as in it can accomplish both in reverse security (The repudiated client can't unscramble any new ciphertext that requires the denied credit to decode) and forward security (The recently joined client can likewise unscramble the beforehand distributed ciphertexts1, in the event that it has adequate qualities). Our plan does not require the server to be completely 
trusted, in light of the fact that the key refresh is authorized by each characteristic specialist not the server. Regardless of the possibility that the server is not semi-confided in a few situations, our plan can at present assurance the retrogressive security. At that point, we apply our proposed revocable multi-expert $\mathrm{CP}-\mathrm{ABE}$ plot as the basic strategies to develop the expressive and secure information get to control conspire for multiauthority distributed storage frameworks [1], [5].

\section{Existing System}

Single Authority Cipher content Policy Attribute Based encryption, Here there exist just a single specialist which gives credits to numerous clients. And every one of the properties are overseen by this specialist as it were. This delivered a security issue and overhead to the expert as every one of the clients should be kept up and overseen by this specialist as it were. It was not productive as well [1], [2].

Multi-Authority Ciphertext-Policy Attribute Based encryption Here numerous experts exist in the framework every one of the specialists are incorporated into the conveyance of the ascribes to the clients. This plan is more suitable for information get to control of distributed storage frameworks, as clients may hold qualities issued by numerous specialists and information proprietor can share the information utilizing access strategies characterized on the properties by various experts. This diminished the overhead of keeping up various clients. Multi-specialist CP-ABE plot spoke to trait denial issue. Characteristic Revocation As numerous experts exist there will be various ascribes to the client and the properties can be changed powerfully. That is a client can be given some new properties by the expert or repudiated some current traits. This sort of quality disavowal ought to be considered as needs be. The new plan conquers the issue of disavowal yet at the same time there exist security issues in the current framework.

\section{Proposed System:-}

The proposed framework conquers the issue exist in the current framework. We proposed another calculation named as Improved Security information Access Control. This calculation enhances the security of the framework. The information proprietor when stores the information into the cloud server he encodes it and afterward stores it. The keys will be given to the approved clients by regarded specialists. So when the client tries to get to the information to which he is not having the qualified trait the demand gets rejected and the client gets hindered by the specialist. What's more, expert will likewise create a message about the assault to the information proprietor. So that information proprietor can make additionally move. In the event that the client has done it by error the approved client can contact the information proprietor to unblock him. On the off chance that the client has not done it then additionally the client can contact the information proprietor and can guarantee greater security by requesting that the information proprietor change the login subtle elements. This new calculation additionally gives information honesty. It advises about the assault by the un-approved client to information proprietor when information proprietor checks about it. That is, the point at which the information proprietor needs to check the records put away on the cloud oftentimes. On the off chance that any alterations are found in the document on the server by any unapproved get to then this calculation advises the information proprietor that the record is not protected, it is changed.

Our system is proposed to do the following:

$\square$ Our framework gives forward and in reverse security as well as gives enhanced security by giving access control on approved clients.

The calculation proposed by us enhances the security by advising about the assault to the information proprietor.

We likewise gave the information trustworthiness. As the information proprietor comes to think about the confirmation in the information put away when he checks it.

\section{Implementation}

Module Description:-

1) System Initialization

2) Key Generator 
3) Data encryption by Owners-

4) Data encryption by Users

5) Intrusion alert

6) Attribute Revocation

\section{1)System Initialization}

We view the server as semi trusted, i.e., . That implies the server will attempt to discover however much mystery data in the put away BR documents as could be expected, yet they will genuinely take after the convention when all is said in done. Then again, a few clients will likewise attempt to get to the documents past their benefits. For instance, a drug store might need to acquire the medicines of patients for advertising and boosting its benefits. To do as such, they may connive with different clients, or even with the server. What's more, we accept each gathering in our framework is preloaded with an open/private key match, and element verification should be possible by conventional test reaction conventions [1]. also, not as an autonomous record. Kindly don't reexamine any of the present assignments.

\section{2) Key Generator}

The Key Generator used to create the key for encryption in light of accessible favored systems. AES will create smaller keys with the extra advantage that the cryptosystem is not loaded with patent consistence. Nonetheless, ought to a parallel tumble to figuring out, the key will progress toward becoming traded off (note that AES is a Symmetric Cipher - not an Asymmetric Cipher which has Public and Private Keys). At present, there are three FIPS (Federal Information Processing Standards) endorsed symmetric encryption calculations: AES, Triple DES, and Skipjack. This article will utilize AES or the Advanced Encryption Standard in CBC Mode [3], [1]. Take note of that DES (FIPS 46-3) was pulled back in May 2005, and is never again affirmed for Federal utilize. AES (or Rijndeal - articulated "Rhine dahl") is the work of Joan Daemen and Vincent Rijmen - consequently the portmanteau Rijndael. AES is a 128 piece square figure that acknowledges key lengths of 128 , 192, and 256 bits. The required number of rounds (i.e., direct and non-straight changes), rely on upon the key size [4], [3]. The following are the FIPS 197 conformant Key - Block Round-Combinations. Taking from FIPS 197: For both its Cipher and Inverse Cipher, the AES calculation utilizes a round capacity that is made out of four distinctive byteoriented changes: 1) Byte substitution utilizing a substitution table (S-box), 2) Shifting lines of the State exhibit by various counterbalances, 3) Mixing the information inside every segment of the State cluster, and 4) Adding a Round Key to the State.

\section{3) Data encryption by Owners-}

The primary objective of our system is to give secure client driven BR get to and productive key administration in the meantime. The key thought is to isolate the framework into numerous security spaces (to be specific, open areas (PUDs) and individual spaces (PSDs) as indicated by the distinctive clients' information get to prerequisites. The PUDs comprise of clients who make get to in view of their expert parts, for example, specialists, attendants and restorative analysts. Practically speaking, a PUD can be mapped to an autonomous segment in the general public, for example, the medicinal services, government or protection area. For each PSD, its clients are actually connected with an information proprietor, (for example, relatives or dear companions), and they make gets to BRs in view of get to rights allotted by the proprietor.

\section{4) Data decryption by Users}

In our structure, there are different SDs, various proprietors, numerous AAs, and various clients. Moreover, two ABE frameworks are included. We term the clients having perused and compose access as information perusers and supporters, separately. The proprietors transfer ABEscrambled BR documents to the server. Every proprietor's BR document is encoded both under a specific fine grained and part based get to arrangement for clients from the PUD to get to, and under a chose set of information qualities that permits access from clients in the PSD [9], [7]. Just approved clients can decode the BR records, barring the server. 


\section{5) Intrusion alert}

In proposed framework, interruption ready framework assumes significant liability to alarm mysterious get to string to applicable client/Authority control. The module has very much outlined rich UI to acquire the number endeavor and it will expect string when the endeavor surpass greatest trial endeavour [7], [8], [9]. The endeavor will be included as one of attempt client login/information get to prepare even in the event that if any wrong passage by validated client. Confirmation procedure of information proprietor and client will be logged by cloud server lumberjack. For any client/client account, account status will be crippled by setting status banner of client get to control list. Login endeavor and host points of interest will be signed in non-trustable host list. Choice about get to host is made by getting and dissect about its authentication status [6]. Usually trusted gatherings will have constantly approved mark in testament.

\section{6) Attribute Revocation}

Client status is repudiated to before state, when it is impaired by human blunder or in utilized by others. Client renouncement is handled in two phases. In initial step, debilitated/noted client will get message about its validation bomb on permitted trines. Client needs to check their character to demonstrate the uniqueness. Once the character is affirmed new get to code or mystery key will be send by information proprietor from the server. client needs to demonstrate some record data about their record to distinguish the present client is trustable. New mystery key will be produced cloud server and forward to client.

\section{System Architecture}

A revocable multi-specialist $\mathrm{CP}-\mathrm{ABE}$ plan, to take care of the characteristic repudiation issue in the framework. This strategy is an effective and secure repudiation technique. The trait disavowal technique can productively accomplish both forward security and in reverse security

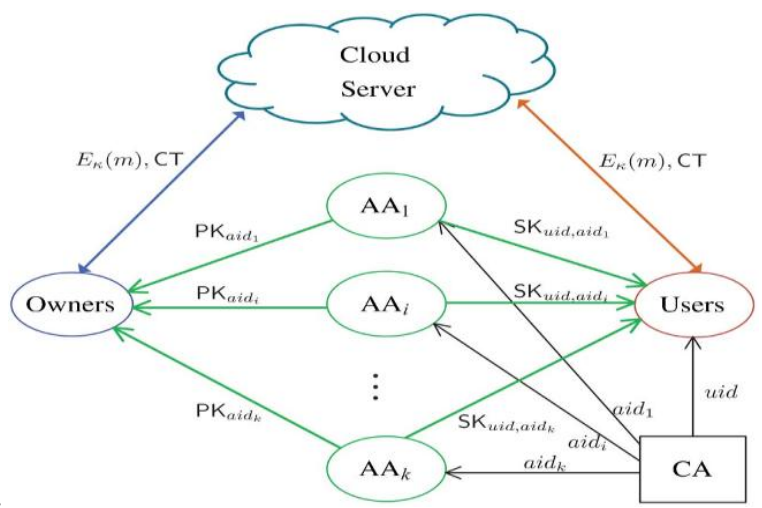

Fig 2: System model of data access control in multi-authority cloud storage

In reverse security conspire the renounced client can't unscramble any new Cipher message that requires the repudiated ascribe to decode. In Forward security the recently joined client can likewise decode the already distributed figure writings, in the event that it has adequate traits. In addition, while refreshing the figure messages, every one of the clients need to hold just the most recent mystery key, as opposed to keep records on all the past mystery keys. We consider an information get to control framework in multi-specialist distributed storage, as portrayed in Figure1. There are five sorts of elements in the framework: a testament expert (CA), property specialists (AAs), information (proprietors), the cloud (server) and information buyers (clients) [5], [7].

\section{Our Data Access Control Scheme}

In this area, we initially give a diagram of the difficulties and methods. At that point, we propose the nitty gritty development of our get to control plot which comprises of five stages: System Initialization, Key Generation, Data Encryption, Data Decryption and Attribute Revocation. To outline the information get to control plot for multi-specialist distributed storage frameworks, the primary testing issue is to build the fundamental Revocable Multi-expert HABEprotocol. In Chase proposed a multi-specialist HABEprotocol, be that as it may, it can't be straightforwardly connected 
as the hidden procedures due to two principle reasons: 1) Security Issue: Chase's multi-expert CPABE convention enables the focal expert to unscramble all the figure writings, since it holds the ace key of the framework. 2) Revocation Issue: Chase's convention does not bolster property disavowal. We propose another revocable multi-expert HABEprotocol in view of the single-specialist CPABE proposed by Lewko and Waters . That is we stretch out it to multi-specialist situation and make it revocable. We apply the procedures in Chase's multi-expert HABEprotocol to entwine the mystery keys produced by various specialists for a similar client and keep the arrangement assault. In particular, we isolate the usefulness of the expert into a worldwide authentication specialist (CA) and various quality specialists (AAs). The CA sets up the framework and acknowledges the enlistment of clients and AAs in the framework. It allots a worldwide client personality uid to every client and a worldwide specialist character help to each trait expert in the framework. Since the uid is internationally one of a kind in the framework, mystery keys issued by various AAs for the same uid can be entwined for unscrambling. Additionally, on the grounds that every AA is related with a guide, each characteristic is recognizable despite the fact that a few AAs may issue a similar quality. To manage the security issue, rather than utilizing the framework one of a kind open key (created by the remarkable ace key) to scramble information, our plan requires all credit specialists to produce their own open keys and uses them to encode information together with the worldwide open parameters. This keep the endorsement expert in our plan from decoding the figure writings. To take care of the characteristic repudiation issue, we appoint a rendition number for each quality. At the point when a property renouncement happens, just those segments related with the disavowed characteristic in mystery keys and figure writings should be refreshed. At the point when a quality of a client is denied from its comparing AA, the AA creates another variant key for this renounced trait and produces a refresh key. With the refresh key, every one of the clients, aside from the disavowed client, who hold the repudiated traits can refresh its mystery key (Backward Security). By utilizing the refresh key, the parts related with the disavowed quality in the figure content can likewise be refreshed to the present form. To enhance the effectiveness, we appoint the work heap of figure content refresh to the server by utilizing the intermediary reencryption technique, to such an extent that the recently joined client is additionally ready to unscramble the already distributed information, which are encoded with the past open keys, in the event that they have adequate traits (Forward Security). Additionally, by refreshing the figure messages, every one of the clients need to hold just the most recent mystery key, as opposed to keep records on all the past mystery keys [1].

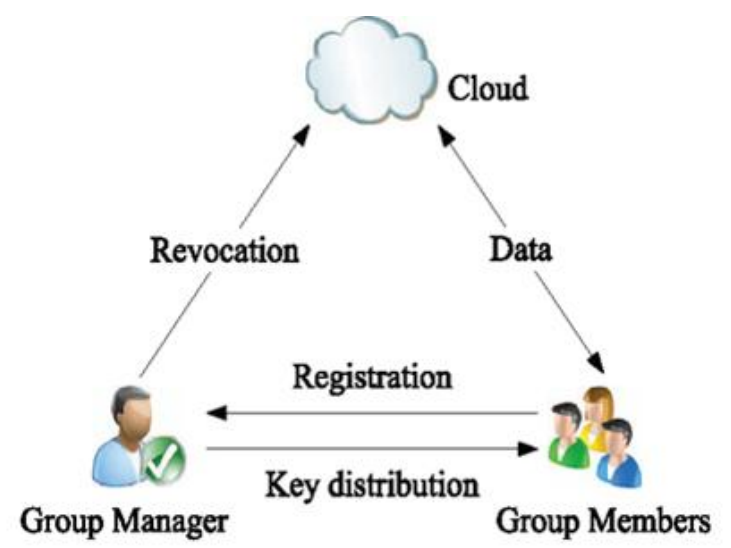

Fig 3 : Expressive, Efficient, and Revocable Data Access Control for Multi-Authority Cloud Storage

\section{Conclusion}

In this paper, we proposed a revocable multi-authority CPABE scheme that can support efficient attribute revocation. Then, we constructed an effective data access control scheme for multiauthority cloud storage systems. We also proved that our scheme was provable secure in the random oracle model [2], [3]. 


\section{References}

[1] Chase and S.S.M. Chow, "Improving Privacy and Security in Multi-Authority Attribute-Based Encryption," in Proc. 16th ACM Conf. Computer and Comm. Security (CCS’09), 2009, pp. 121-130.

[2] A.B. Lewko and B. Waters, "Decentralizing Attribute-Based Encryption," in Proc. Advances in CryptologyEUROCRYPT’11, 2011, pp. 568-588.

[3] S. Yu, C. Wang, K. Ren, and W. Lou, "Attribute Based Data Sharing with Attribute Revocation," in Proc. 5th ACM Symp. Information, Computer and Comm. Security (ASIACCS'10), 2010, pp. 261- 270.

[4] M. Li, S. Yu, Y. Zheng, K. Ren, andW. Lou, "Scalable and Secure Sharing of Personal Health Records in Cloud Computing Using Attribute-Based Encryption," IEEE Trans. Parallel Distributed Systems, vol. 24, no. 1, pp. 131143, Jan. 2013

[5] J. Hur and D.K. Noh, "Attribute-Based Access Control with Efficient Revocation in Data Outsourcing Systems," IEEE Trans. Parallel Distributed Systems, vol. 22, no. 7, pp. 1214-1221, July 2011.

[6] S. Jahid, P. Mittal, and N. Borisov, "Easier: Encryption-Based Access Control in Social Networks with Efficient Revocation,' in Proc. 6th ACM Symp. Information, Computer and Comm. Security (ASIACCS'11), 2011, pp. 411415.

[7] S. Ruj, A. Nayak, and I. Stojmenovic, "DACC: Distributed Access Control in Clouds," in Proc. 10th IEEE Int'l Conf. TrustCom, 2011, pp. 91-98.

[8] K. Yang and X. Jia, “Attribute-Based Access Control for Multi-Authority Systems in Cloud Storage," in Proc. 32th IEEE Int'l Conf. Distributed Computing Systems (ICDCS'12), 2012, pp. 1-10.

[9] Boneh and M.K. Franklin, "IdentityBased Encryption from the Weil Pairing," in Proc. 21st Ann. Int'l Cryptology Conf.: Advances in Cryptology - CRYPTO’01, 2001, pp. 213-229.

[10] Gopi Krishna Battula, M Tech, LBRCE, Under TheGuidence Of B. Siva Rama Krishna (Asst. Professor)

IOSR Journal of Computer Engineering (IOSR-JCE) is UGC approved Journal with Sl. No. 5019, Journal no. 49102.

* Mr. B. Gopi Krishna. "Hierarchical Attribute Based Revocable Data Access Control For Multi Authority Cloud Storage." IOSR Journal of Computer Engineering (IOSR-JCE) 19.4 (2017): 91-97. 